\title{
Potential-energy Surfaces, Unimolecular Processes and Spectroscopy
}

\author{
Rudolph A. Marcus \\ Noyes Laboratory of Chemical Physics, California Institute of Technology, Pasadena, \\ California 91125, U.S.A.
}

\begin{abstract}
The present symposium brings together research in a number of fields: the quantum-chemical calculation of molecular potential-energy surfaces, rotational-vibrational spectroscopy, methods of calculating rotational-vibrational energy levels, unimolecular reactions and intramolecular dynamics. Several aspects of the work are discussed, including some recent developments on rates and products' quantum state distributions for unimolecular dissociations having highly flexible transition states. The usefulness of having improved potential-energy surfaces, particularly the bonding and hindered rotational potentials in the dissociations, is noted. In various other studies in this symposium a better knowledge of the surfaces would be particularly helpful. New results on a semiclassical quantization method are also described.
\end{abstract}

\section{Potential-energy Surfaces, Spectroscopy and Dynamics}

The papers of this Faraday Symposium illuminate particularly the interaction of research in several fields. These areas include theoretical calculations of molecular potentialenergy surfaces, the spectroscopic study of intramolecular motion, and experimental and theoretical studies of unimolecular processes and of intramolecular dynamics. Each area has undergone significant recent developments, and the increasingly detailed information that is now becoming available for each should further enhance this interaction. The organizers of this symposium are to be congratulated for the timeliness of their central theme.

The relation between the theoretical study of molecular potential-energy surfaces (p.e.s.) and the experimental spectroscopic investigation of molecules has, of course, a long history. The recent advances made by quantum chemists on calculations of the p.e.s. are noted in this symposium by Amos et al. ${ }^{1}$ Several of these papers provide examples of the $a b$ initio calculation of these surfaces. ${ }^{2,3}$ To make a connection between such calculations and spectroscopic experimental data, a calculation of the rotationalvibrational molecular energy levels based on these surfaces is required and is discussed here in a number of articles. ${ }^{1-7}$ These include studies at higher energies where perturbation-type approaches have become inadequate.

In an alternative procedure, also represented by a number of papers in this conference, ${ }^{7-12}$ parameters of the p.e.s. such as anharmonicity constants and parameters such as Coriolis constants are estimated, instead, by a fit to the spectroscopic data. Lehmann and McCoy, ${ }^{10}$ for example, describe in some detail the fitting of the $\mathrm{NH}_{3}$ spectrum to an effective Hamiltonian containing various resonance terms.

Theoretical studies of the dynamics of intermolecular and intramolecular energy redistribution benefit from a detailed knowledge of these potential-energy surfaces. A variety of such problems is treated in the present set of papers. ${ }^{13-18}$ Treatments of chemical reactions also benefit and involve regions of the p.e.s. where the anharmonicity is considerable. Such regions of coordinate space are only beginning to be observable 
by spectroscopic means. Examples occur in recent studies of a molecule in the process of falling apart, using picosecond and femtosecond real-time measurements ${ }^{19,20}$ and high-resolution Raman-type scattering. ${ }^{21}$ Interesting results have been found, including a time delay ${ }^{19}$ in a reaction within a van der Waals' complex, $\mathrm{CO}_{2}-\mathrm{HI}^{*} \rightarrow \mathrm{CO}_{2} \mathrm{H}+\mathrm{I}$ and, in a $\mathrm{CH}_{3} \mathrm{I}$ photodissociation, the coupling of the $\mathrm{H}_{3} \mathrm{C}-\mathrm{I}$ dissociating bond with the $\mathrm{CH}_{3}$ umbrella motion. ${ }^{21}$ The products' quantum state distribution is also known in the former reaction for the case where the photoexcited $\mathrm{Hi}^{*}$ is replaced by a $\mathrm{HBr}^{*} .^{22}$ In the region of coordinate space important to unimolecular dissociations the main source of information thus far has been quantum-chemical calculations but, as we shall note later, such calculations are presently rather sparse in an important region. In each of the above experiments ${ }^{19-21}$ two p.e.s. are involved in the optical observations, and a detailed knowledge of these p.e.s. is useful.

Studies of the nature of highly excited vibrational states include those by stimulated emission pumping, ${ }^{23}$ e.g. for molecules such as $\mathrm{C}_{2} \mathrm{H}_{2}$ and $\mathrm{CH}_{2} \mathrm{O}$. They are represented here by the work of Temps et al., ${ }^{14}$ who consider collisional state-to-state processes and find propensity rules reflecting long-range dipole-dipole interactions (large collision cross-sections) between two $\mathrm{CH}_{2} \mathrm{O}$ molecules. The excitation of $\mathrm{CH}$ and $\mathrm{OH}$ overtones has provided results on energy-resolved unimolecular rate constants and spectroscopy of excited local modes. One recent advance, the use of a 'two-colour' excitation, leading to a 'vibrationally mediated dissociation', is described here by Likar et al. ${ }^{18}$ Until this advance was made, dissociation via a lower overtone could only occur in a thermally assisted way, a way which entails a non-monochromatic energy distribution of the reacting molecule and hence involves a more complicated interpretation. In this symposium Lehmann and Coy describe a new 'microwave-detected, microwave double resonance' technique for the study of the energy levels of $\mathrm{NH}_{3}{ }^{10}$ Baggott et al. ${ }^{8}$ and Likar et al. ${ }^{18}$ discuss the overtone spectra. Narrow spectra have been observed for the third $\mathrm{CH}$ overtone in benzene by Page et al., ${ }^{24 a}$ a factor of $c a .10$ narrower than that previously found both experimentally ${ }^{24 b}$ and in classical trajectory studies. ${ }^{24 c}$ This previous experimental work was apparently complicated by inhomogeneous line broadening, as had been suspected. We return to this point later.

There are, of course, several spectroscopic studies not represented in this symposium (it would hardly be possible to represent them all!). In the frequency domain they include sub-Doppler studies of vibronic couplings in electronically excited benzene, ${ }^{25}$ and the observation of vibrational quantum beats in molecules such as anthracene. ${ }^{26}$

Most theoretical studies of rotational-vibrational spectra have been primarily concerned with molecules at comparatively low energies. In studies at higher energies theoretical investigations of the spectral features have often been based on model Hamiltonians containing only a few (two or three) coordinates. These include the treatment of the $\mathrm{CH}$ stretch-doubly degenerate $\mathrm{HCX}$ bend in a 2:1 Fermi resonance in $\mathrm{CHX}_{3}$ molecules by Voth et al., ${ }^{27}$ Moore and coworkers, ${ }^{28}$ and Halonen et al., ${ }^{7}$ all using curvilinear coordinates, and earlier studies by Quack and coworkers using rectilinear coordinates. ${ }^{29}$ A concise description of the advantages of the curvilinear ones for high $\mathrm{CH}$ overtones is given here by Halonen et al. ${ }^{7}$ In this use of curvilinear coordinates, Voth et al. found (for $\mathrm{X}=\mathrm{D}$ ) that a purely kinetic-energy coupling term, sometimes used in other work, was inadequate to represent the spectroscopic results for the $2: 1$ resonance, and that a potential-energy coupling term was also needed. ${ }^{27}$ These results further emphasize the need for obtaining a reliable p.e.s. A similar finding on the importance of the potentialenergy term is reported in the present symposium by Halonen et al. ${ }^{7}$ for $\mathrm{X}=\mathrm{D}$ and $\mathrm{F}$.

In this three-coordinate $\mathrm{CH}$ stretch-doubly degenerate $\mathrm{HCX}$ bend problem the vibrational angular momentum $l$ of the bend is frequently assumed to be a constant of the motion. In the quantum case this approximation permits, of course, the Hamiltonian matrix to be block-diagonalized, while in the classical case it reduces the three-coordinate problem to a two-coordinate one when the appropriate variables are used. (E.g. in 
action-angle coordinates, if $l$ is a constant of the motion the Hamiltonian does not depend on its conjugate coordinate. The classical Hamilton's equations of motion may then be integrated, $l$ being a constant, using the two remaining coordinates and their conjugate momenta.)

Other articles on these few-coordinate treatments in this symposium include that by Baggott et $a l^{8}$ on high $\mathrm{CH}$ overtones in $\mathrm{HCN}$, in which there is an interaction of two anharmonic bond-stretching modes. Using a complex coordinate method Hutchison and Marshall ${ }^{15}$ treat the coupling of a $\mathrm{CH}$ overtone and a $\mathrm{CC}$ bond. A coupling between a ring-vibrational mode and a torsional mode in $p$-flourotoluene is described by Longfellow and Parmenter, ${ }^{16}$ who also note the resolved spectroscopic data now becoming available. $^{30}$ They also discuss the role of this coupling in intramolecular vibrational energy redistribution (IVR). ${ }^{16}$ A treatment of three interacting symmetric modes in $\mathrm{NH}_{3}^{+}$ is given by Botschwina. ${ }^{2}$ The usefulness of few-coordinate models for treating the positions of $\mathrm{CH}$ overtone lines in, for example, $\mathrm{HCN}$ (Baggott et al. here ${ }^{8}$ ), $\mathrm{HCX}_{3}$ (Halonen et al..$^{7}$ and others ${ }^{27-29}$ ) and $\mathrm{C}_{2} \mathrm{H}_{2},{ }^{31}$ is noted by Lehmann and Coy. ${ }^{10} \mathrm{An}$ in-plane classical-mechanical model, introduced to look for a progression seen in low resolution for $\mathrm{H}_{3}^{+}$, is described by Chambers et al., ${ }^{13}$ who also discuss the interesting 'weakly chaotic' features of the classical-mechanical motion.

A variety of other approximate treatments of the molecular motion are described in this symposium, sometimes to treat a larger number of coordinates. One such approach for calculating the energy levels of a several-coordinate system involves an adiabatic approximation, in which the variables are divided into 'fast' and 'slow', this classification being capable of extension to different degrees of 'slowness'. In a treatment of $\mathrm{C}_{2} \mathrm{H}_{2}$ here by Amos et al., ', all of the vibrations are included, the 'fastest' coordinates being the two $\mathrm{CH}$ stretches and the slowest the torsional angle between the $\mathrm{HCC}$ and $\mathrm{CCH}$ planes. Halonen et al. ${ }^{7}$ use both an adiabatic and a separable basis set in their treatment of the $\mathrm{CH}$ stretch-doubly degenerate $\mathrm{CHX}$ bend Fermi resonance, and compare results obtained from an $a b$ initio p.e.s. and from experiment, by fitting to an effective tridiagonal form of Hamiltonian matrix. Floppy molecules are discussed by Jensen ${ }^{6}$ using a 'non-rigid bender' plus Morse-stretch Hamiltonian for triatomics, which is applied to $\mathrm{H}_{3}^{+}$and to its isotopes. Rotational-vibrational coupling for states with relatively high angular momentum quantum numbers $J$ are treated by Tennyson et al. ${ }^{4}$ using a two-step variational procedure. They apply it to isotopes of $\mathrm{H}_{3}^{+}$and to a van der Waals' complex, and describe the problems arising at high $J$.

Another approximate procedure, a self-consistent field calculation ${ }^{32}$ of ro-vibrational energy levels, is applied here by Carney to the lower vibrational states of $\mathrm{H}_{3}^{+}$-type molecules. ${ }^{3}$ Semiclassical evaluation of vibrational Hamiltonian matrix elements introduced by Gerber and Ratner ${ }^{32}$ and that of other matrix elements, such as that of the dipole operator by other authors ${ }^{33}$ is employed here. ${ }^{3}$ Some comparison of the relative merits of these many approaches, of others described in the symposium [e.g. ref. (5)] and of one described below, might be a useful topic for discussion. As one might see from the above survey the spectroscopy ${ }^{34}$ of the molecular ion $\mathrm{H}_{3}^{+}$has aroused particular interest.

An interesting semiclassical method for obtaining the vibrational energy levels of triatomic molecules has recently been described elsewhere by Noid and coworkers, ${ }^{35,36}$ and I should like to comment on it here. These authors proceed from an analytical potential-energy surface or from a numerically given surface using a spline fit, together with expressions for the partial derivatives for use in integrating the classical equations of motion, calculate the Poincaré surfaces of section (S.O.S.) for these three-vibrational coordinate systems by interpolation, ${ }^{36}$ and by this means quantize the system semiclassically. Over the years the Poincare S.O.S. method for semiclassical quantization ${ }^{37}$ has proved to be reliable and straightforward. (A recent comparison with other methods has been given for systems containing resonances. ${ }^{38}$ ) One interesting feature is that now 
only one trajectory or less is needed per eigenvalue for the three-coordinate system, ${ }^{36}$ and only $\mathrm{ca}$. one per three eigenvalues for two-coordinate studies. ${ }^{35}$ These results are a striking improvement over the early days ${ }^{37,39}$ when we used for the two-coordinate systems $c a$. six trajectories per eigenvalue, together with some iterative process.

It would be desirable, of course, to combine this apparently straightforward method of Noid's with a treatment of the rotations, perhaps with one such as that of Tennyson et al. ${ }^{4}$ and so treat the full ro-vibrational energy levels, even at high energies. Limitations on the method occur, when the relevant trajectories become chaotic. If appreciable accuracy is desired this behaviour is a limitation of all semiclassical trajectory methods. However, Noid and coworkers ${ }^{40}$ have also discussed and applied a power spectral method which uses only very short-time trajectories (a fraction of a vibrational period) and it may alleviate this difficulty somewhat, at least for weakly chaotic trajectories. An example of the latter is given in this symposium by Chambers et al. ${ }^{13}$ in their classical model for $\mathbf{H}_{3}^{+}$. The usual power spectral trajectory method ${ }^{33}$ requires, instead, many vibrational periods, but in other respects is complementary to the new method. A nice feature of semiclassical methods is that they can be used with arbitrary potentials. To be effective the purely quantum methods must be such that the matrix elements can be efficiently evaluated, and so frequently resort is made to a polynomial expansion of the potential. Brodersen comments on that expansion for molecules containing hydrogen. ${ }^{6}$

\section{Unimolecular Reactions and Potential-energy Surfaces}

With the introduction of short-pulse laser techniques, ${ }^{41}$ time-resolved measurements have permitted the direct determination of energy-resolved ${ }^{41}$ and low-angularmomentum energy-resolved ${ }^{42}$ rate constants for unimolecular dissociations. An example of a 'two-colour' photodissociation study is given in this symposium, as already noted, by Likar et al. ${ }^{15}$ Numerous other studies (infrared multiphoton dissociation, ${ }^{43}$ molecularbeam studies of product translational energy distribution, ${ }^{44}$ infrared chemiluminescence of vibrational distributions ${ }^{45}$ ) have been made, and have contributed to the richness of data for comparison with unimolecular reaction rate theory. An example of a laserinduced fluorescence ${ }^{46}$ determination of the quantum state distribution of the reaction products is also given here. ${ }^{18}$ An intramolecular $\mathrm{H}$-atom transfer is treated in the present symposium by Ruf and Miller, ${ }^{47}$ and represents an interesting class of reactions.

On the theoretical side it is now practical to make RRKM-type calculations of energy-resolved unimolecular and angular momentum-resolved rate constants $k_{E J}$ for a case frequently encountered in a dissociation, namely where the transition state is rather flexible. ${ }^{48}$ (In earlier times it was practical to treat mainly 'loose' and 'tight' transition states.) In a flexible transition state, the partially hindered rotations of the incipient product fragments can be highly coupled to each other and to the overall rotations. The part of the potential-energy surface which plays a role in this transition state depends upon the total energy $E_{.}^{48}$

We recall briefly this variationally implemented RRKM theory both for the rates ${ }^{48}$ and now for the products' quantum state distribution. ${ }^{49}$ The unimolecular rate constant $k_{E J}$ at a particular total energy $E$ and total angular momentum quantum number $J$ is given by ${ }^{48}$

$$
k_{E J}=\frac{N_{E J}\left(R^{\ddagger}\right)}{h \rho_{E J}}
$$

where $N_{E J}(R)$ is the number of quantum states of the dissociating molecule as a function of any selected reaction coordinate $R$, and $N_{E J}\left(R^{*}\right)$ is obtained variationally by finding the minimum value of $N_{E J}(R)$ as a function of $R$, thereby defining $R^{\ddagger} ; \rho_{E J}$ is the density of ro-vibrational states of the molecule for the given $E$ and $J . N_{E J}(R)$ is evaluated for 
these highly flexible transition states, after classifying the modes of motion of the dissociating molecules as 'conserved modes' and 'transitional modes', ${ }^{48}$ the former being those which do not undergo a major change in the nature of their motion on going from the reactant to the separated fragments, while the 'transitional modes' do. The latter typically consist of the bending motions which become rotations of the fragments and of these overall rotations, to which these modes are coupled. When the effect of total angular momentum conservation is taken into account there remain normally six transitional modes for the case of two non-linear polyatomic fragments. ${ }^{48} N_{E J}(R)$ is given by a convolution ${ }^{48}$

$$
N_{E J}(R)=\int_{0}^{E} N_{v}(E-\varepsilon) \Omega_{J}(\varepsilon) \mathrm{d} \varepsilon
$$

where $N_{v}(E-\varepsilon)$ is the number of quantum states of the conserved modes with an energy less than or equal to $E-\varepsilon$ and $\Omega_{J}(\varepsilon)$ is the density of states of the transitional modes at an energy $\varepsilon$.

In order to determine $N_{E J}(R)$ in the transition-state region, $\Omega_{J}(\varepsilon)$ is needed, which, in turn, requires some knowledge of the potential-energy surface (particularly the bonding potential and the potential hindering the rotation of the fragments in the vicinity of $R^{\ddagger}$ ). The integral in eqn (2) is evaluated by a Monte Carlo calculation. Several recent studies applying the theory to thermal and to energy-resolved rate constants have been made. ${ }^{48,50}$

At low $E$ values $R^{\ddagger}$ is large and the calculation of the hindering potential for the full solid-angle range for the fragment rotations is needed. With increasing energy $E$, $R^{\ddagger}$ tends to move to shorter fragment-fragment separation distances, ${ }^{48,50-52}$ where the fragment rotations eventually become librations, and then a knowledge of the p.e.s. is needed in this region also. It seems clear that there is a real opportunity for fruitful interaction between quantum chemists who can calculate the potential-energy surfaces and those interested in applying such results to understand or predict the rate constants and the products' quantum state distributions. A first estimate for $R^{\ddagger}(E)$ can be made using a rather approximate p.e.s. in the theory for $k_{E J}$, and an improved surface can then be obtained in this region of $R^{\ddagger}$ using more accurate quantum-chemical calculations.

Evidence for the trend of $R^{\ddagger}(E)$ to decrease with increasing $E$ is found from the behaviour of the rate constants themselves. ${ }^{48,50-52}$ It would be interesting to see if other evidence can also be obtained. One such possibility is suggested in the work of Likar et $a l^{18}$ in this symposium. In their study of the photodissociation of $\mathrm{HONO}_{2}$ they consider the ratio of populations of the lambda doublet states of $\mathrm{OH}$ and the influence regarding the geometry throughout the dissociation. It will be interesting to study the ratio at low $E$, where deviation from planarity ( $R^{\dagger}$ becomes larger) may be large. A variety of effects can occur here, and the experiments at low $E$ will be interesting.

The experimental determination of the quantum state distribution of the products of unimolecular dissociations has been of particular interest. ${ }^{18,53-55}$ Particularly at energies insufficient to excite the reaction products vibrationally there has been good agreement between these distributions and the results predicted from phase-space theory (PST) in some recent studies. ${ }^{53,55}$ Since in PST only the long-range potential is utilized, a knowledge of the detailed p.e.s. is not needed! However, deviations from PST appear at higher energies. ${ }^{53}$ Deviations from PST have also been noted for the translational energy distribution of the products in the unimolecular dissociation of ions. ${ }^{56}$

It is of particular interest that PST is successful for predicting the products' internal state distribution at low energies, but that it shows significant deviations for the values of the thermally averaged rate constants. ${ }^{57}$ Of course, the typical energy domain sampled in the two types of experiment can differ.

Recently, we formulated a theory ${ }^{49}$ designed to treat both the rates and the quantum state distribution of the products. The rates were calculated using eqn (1) and (2) as 
before. For the quantum state distribution of the products, we assumed that the conserved modes behave adiabatically from $R^{\ddagger}$ to $\infty$, but that the transitional modes behaved non-adiabatically in the region from $R^{*}$ to the 'loose transition-state' region. ${ }^{49}$ (The latter modes tend to be low-frequency modes near $R^{t}$ and so are expected to interchange their energy relatively easily during the separation, much as molecular rotations do in a short-range collision.) The theoretical results for the products' internal state distribution $^{49}$ reduce to those of PST in the sub-vibrational excitation region, but show deviations from PST at higher energies. In contrast to the above assumption, it is assumed in the ${ }^{6}$ adiabatic channel model ${ }^{58}$ that all coordinates behave adiabatically in the exit channel.

The RRKM rate constants $k_{E J}$ [eqn (1)] are typically less than those of PST, since the latter neglects the effects of restricted rotation of the fragments in the transition state. However, at sufficiently low energies, the transition state typically moves, as already noted, to larger fragment-fragment separation distances, and then the RRKM $k_{E J}$ tends to approach the PST limit [see ref. (52) for a more detailed discussion of this point]. Extensive data on rates and on products' quantum state distributions are becoming available as a function of the energy $E$, and a knowledge of the p.e.s. in the important regions, given by $R^{\ddagger}(E)$, will be very helpful in this field.

Particularly noteworthy too, I believe, are the differences found in the products' quantum state distribution when the photodissociation is via a u.v. excitation to a purely repulsive state, followed immediately by dissociation, as compared with excitation to a longer-lived state. The considerable differences in the products' quantum state distribution are discussed by Likar et al. ${ }^{18}$ The time available for intramolecular energy transfer is much less in the direct dissociation case. Further comparisons along these lines, together with the work referred to earlier on the direct dissociations, ${ }^{19-21}$ can be expected to be a source of information on IVR.

\section{Intramolecular Dynamics}

In the treatment of intramolecular dynamics in actual molecules, a substantial number of coordinates can be expected to be involved, and their number poses a major challenge. Lehmann and $\mathrm{Coy}^{10}$ describe the time-evolution of an excited local-mode NH state, using their five-coordinate model. Some insight into IVR has been obtained from examination of individual two-coordinate resonances, a point illustrated here by Hutchison and Marshall. ${ }^{15}$ Many authors have considered such resonances as a mechanism for intramolecular energy transfer. What is particularly desirable now is the development of methods capable of treating molecules with all the vibrational and rotational coordinates. One possibility is the RRGM method, ${ }^{59}$ which is applied here ${ }^{15}$ to treat a model system containing many states, intended to simulate a complex molecule. Other methods which have been or are being developed to treat this intramolecular vibrational energy redistribution include a partitioning-type method, ${ }^{60}$ a generalized moment method for mean relaxation times, ${ }^{61}$ a wave-packet method $^{62}$ and an artificial intelligence method. ${ }^{6.3}$ An artificial intelligence method has been used to treat the related problems of multiphoton absorption ${ }^{64}$ and the calculation of eigenvalues. ${ }^{65}$ The artifical intelligence method has been applied to an 11-coordinate IVR problem. ${ }^{63}$

\section{Coordinate Systems}

We have already noted that curvilinear coordinates, ${ }^{1,7}$ as well as rectilinear ones, ${ }^{3}$ have been used in the treatment of vibrational Hamiltonians, both in this symposium and elsewhere. In the field of reaction rates or of related properties, several coordinate systems have found use. The 'natural collision coordinates' which pass smoothly from reactants to products, and the associated Hamiltonian have been used, both in their original form ${ }^{66}$ and in a form (the 'reaction-path Hamiltonian') ${ }^{67}$ which on one hand 
was an extension of the original, in that the reaction-path coordinate coefficient in the kinetic energy could depend on more coordinates, and on the other was an approximation, in its neglect of certain kinetic coupling terms and use of small displacements for motion transverse to the reaction path. Another set of coordinates that has found important use in calculations of reaction rates are the hyperspherical coordinates. ${ }^{68}$ The first such fully three-dimensional calculation was recently reported by Hipes and Kuppermann. ${ }^{68}$ Their use is illustrated in this symposium by Chambers et al. ${ }^{13}$ for an in-plane model of the $\mathrm{H}_{3}^{+}$molecule.

Hyperspherical coordinates have been used earlier to treat $\mathrm{H}$-atom transfer in a triatomic collinear heavy-light-heavy atom system. ${ }^{69-73}$ This use permitted the introduction of a vibrationally adiabatic approximation and led to excellent agreement ${ }^{70-72}$ with numerical quantum-mechanical reaction probabilities for the same model symmetric systems. The results were also extended to nearly degenerate transitions. ${ }^{72,73}$ Another set of coordinates, Cartesian ones, for these systems have been introduced by Ruf and Miller, ${ }^{47}$ to treat an intramolecular $\mathrm{H}$-atom transfer. It will be interesting to compare these results with those obtainable with (or generalizable from) the hyperspherical coordinates which we used earlier. ${ }^{70}$

\section{Chaos, Intramolecular Dynamics and Observables}

One topic that has aroused considerable interest in the chemical physics community, particularly since the early 1970s, and the interest of mathematicians and physicists for a longer period, is the question of regularity of motion in classical mechanics for systems which do not have the simplifying feature of permitting a separation of variables. ${ }^{74}$ The original interest was in questions ranging from the non-statistical distribution of stars in certain galaxies ${ }^{75}$ (their long-time existence notwithstanding) to the questions of stability of orbits of various heavenly bodies, and indeed the long-term stability of the solar system itself. Each of these systems is a non-separable classical-mechanical system.

Concepts such as regularity in the motion ('quasi-periodic' behaviour), the famous Kolmogorov-Arnold-Moser theorem for such motion for small perturbations from separability (extremely small!), and a rigorous result of Sinai on an ergodic system, have been described. ${ }^{74}$ For perturbations that are more than extremely small, the information available has come mainly from numerical integration of the classical equations of motion. Both quasi-periodic trajectories, which move on an $N$-dimensional torus in a $2 \mathrm{~N}$-dimensional phase space (and are therefore highly non-statistical) and chaotic trajectories have been found. Their relative importance depends on the Hamiltonian, the energy and the initial conditions. In turn, from semiclassical theory, much is known about the quantum behaviour of the corresponding systems when the relevant trajectories are quasi-periodic. ${ }^{76}$ An example of a weakly chaotic system, in which the trajectory tends to remain near some 'vague torus' in phase space, ${ }^{77,78}$ before switching to another "vague torus', ${ }^{78}$ is described in this symposium by Chambers et al. ${ }^{13}$ in their calculations on $\mathrm{H}_{3}^{+}$.

The main interest by chemical physicists in the non-separable mechanics and classical trajectories has been many-fold: first, there was the novelty of there being new results in a seemingly mature and well understood field; secondly was the hope that since classical trajectories have been the principal source of relating chemical reaction crosssectional data obtained within molecular beams to potential-energy surfaces, and (with the late Don Bunker and his successors) for examining the statistical or non-statistical behaviour of isolated molecules, a deeper understanding of these aspects might develop from the relatively new ideas; finally, there was the intriguing prospect of searching for a possible quantum-mechanical analogue to these striking classical-mechanical concepts on quasi-periodic and chaotic behaviour (from semiclassical arguments, the quantummechanical behaviour should approach the classical behaviour in the limit of $h \rightarrow 0$ ). 
There has been progress on this classical-quantum relationship, but no rigorous results in general. Indeed, there appears to be no rigorous definition of quantum quasi-periodicity and so none of quantum chaos. There are various ideas, and considerable discussion. Concepts such as Chirikov's overlapping resonances ${ }^{79}$ as a source of classical chaotic behaviour, the relation between the classical-mechanical resonances and some 'avoided crossings' of quantum-mechanical eigenvalues, ${ }^{80}$ and the mixing of states via overlapping avoiding crossings have been discussed, ${ }^{80,81}$ and many related or other ideas abound, e.g. ref. (62) and (82)-(86).

It has also been realized that when the resonances causing some locally chaotic classical behaviour occupy a relatively small region of phase space that is small in relation to $h$, the corresponding quantum system may show no hint of a local chaos. ${ }^{81,83,84}$ Another difference is that in the quantum case there may be, semiclassically speaking, a nuclear tunnelling from one torus to another, unlike the classical case. Some caution is needed, therefore, in drawing conclusions about the quantum behaviour of a molecule from that of the corresponding classical calculation. Indeed, the new data ${ }^{24 a}$ on narrow $\mathrm{CH}$ overtone homogenous linewidths, mentioned earlier, no longer agree with those previously calculated from classical trajectories. ${ }^{24 c}$ The latter agreed with the older linewidths. $^{24 b}$ Some discussion of trajectory calculations for linewidths is given in this symposium. ${ }^{18}$ There has been a considerable discussion of 'quantum chaos' in the literature. Ultimately, when adequate methods have been applied to the quantum treatment of real molecules and of the many coordinates frequently involved, the behaviour of physical observables will presumably become the main goal of the theory. In this case some of the recent debates on criteria for 'quantum chaos' may wane, although the concepts evolved may prove useful in interpreting the more numerical findings.

It is clear from the articles in this symposium that the fields being discussed are rich in their variety, and that the results and the interaction of those in the different fields can be expected to provide many an interesting discussion, both in this conference and in the future.

It is a pleasure to acknowledge the support of this work by a grant from the National Science Foundation.

\section{References}

1 R. D. Amos, J. Gaw, N. C. Handy and S. Carter, J. Chem. Soc., Faraday Trans. 2, 1988, 84, 1247.

2 P. Botschwina, J. Chem. Soc., Faraday Trans. 2, 1988, 84, 1263.

3 G. D. Carney, J. Chem. Soc., Faraday Trans. 2, 1988, 84, 1277.

4 J. Tennyson, S. Miller and B. T. Sutcliffe, J. Chem. Soc., Faraday Trans. 2, 1988, 84, 1295.

5 S. Brodersen, J. Chem. Soc., Faraday Trans. 2, 1988, 84, 1365.

6 P. Jensen, J. Chem. Soc., Faraday Trans. 2, 1988, 84, 1315.

7 L. Halonen, T. Carrington and M. Quack, J. Chem. Soc., Faraday Trans. 2, 1988, 84, 1371.

8 J. E. Baggott, G. L. Caldow and I. M. Mills, J. Chem. Soc., Faraday Trans. 2, 1988, 84, 1407.

9 J. C. Duncan, D. C. McKean, I. Torto, A. Brown and A. M. Ferguson, J. Chem. Soc., Faraday Trans. $2,1988,84,1423$.

10 K. K. Lehmann and S. L. Coy, J Chem. Soc., Faraday Trans. 2, 1988, 84, 1389.

11 M. Winnewisser and M. Birk, J. Chem. Soc., Faraday Trans. 2, 1988, 84, 1341.

12 G. W. Bryant, D. F. Eggers and R. O. Watts, J. Chem. Soc., Faraday Trans. 2, 1988, 84, 1443.

13 A. V. Chambers, M. S. Child and R. Pfeiffer, J. Chem. Soc, Faraday Trans, 2, 1988, 84, 1305.

14 F. T. Temps, S. Halle, P. H. Vaccaro, R. W. Field and J. K. Kinsey, J. Chem. Soc., Faraday Trans. 2, $1988,84,1620$.

15 J. S. Hutchison and K. T. Marshall, J. Chem. Soc., Faraday Trans. 2, 1988, 84, 1535.

16 R. J. Longellow and C. S. Parmenter, J. Chem. Soc., Faraday Trans. 2, 1988, 84, 1499.

17 W. P. Reinhardt and C. Duneczky, J. Chem. Soc., Faraday Trans. 2, 1988, 84, 1511.

18 M. D. Likar, J. E. Baggott, A. Sinha, T. M. Ticich, R. L. Vander Wal and F. F. Crim, J. Chem. Soc., Faraday Trans. 2, 1988, 84, 1483.

19 N. F. Scherer, L. R. Khundkar, R. B. Bernstein and A. H. Zewail, J. Chem. Phys., 1987, 87, 1451.

20 M. Dantus, M. J. Rosker and A. H. Zewail, J. Chem. Phys., 1987, 87, 2395.

21 D. Imre, J. L. Kinsey, A. Sinha and J. Krenos, J. Phys. Chem., 1984, 88, 3956. 
22 G. Radhakrishnan, S. Buelow and C. Wittig, J. Chem. Phys., 1984, 84, 727; S. Buelow, M. Noble, G. Radhakrishnan, H. Reisler and C. Wittig, J. Phys. Chem., 1986, 90, 1015.

23 H. L. Dai, C. L. Korpa, J. L. Kinsey and R. W. Field, J. Chem. Phys., 1985, 82, 1688; E. Abrahamson, R. W. Field, D. Imre, K. K. Innes and J. L. Kinsey, J. Chem. Phys., 1985, 83, 453.

24 (a) R. H. Page, Y. R. Shen and Y. T. Lee, Phys. Rev. Lett., 1987, 59, 1293; J. Chem. Phys., submitted; (b) K. V. Reddy, D. F. Heller and M. J. Berry, J. Chem. Phys., 1982, 76, 2814; (c) D-H. Lu, W. L. Hase and R. J. Wolf, J. Chem. Phys., 1986, 85, 4482; D.H. Lu and W. L. Hase J. Phys. Chem., in press; D. L. Clarke and M. A. Collins, J. Chem. Phys., 1987, 86, 6871.

25 E. Riedle, H. J. Neusser and E. W. Schlag, J. Phys. Chem., 1982, 86, 4847; U. Schubert, E. Riedle and H. J. Neusser, J. Chem. Phys., 1986, 84, 5326.

26 W. R. Lambert, P. M. Felker and A. H. Zewail, J. Chem. Phys., 1984, 81, 2217.

27 G. A. Voth, R. A. Marcus and A. H. Zewail, J. Chem. Phys., 1984, 81, 5494.

28 J. S. Wong, W. H. Green, W. D. Lawrance and C. B. Moore, J. Chem. Phys., 1987, 86, 5994; W. H. Green, W. D. Lawrance and C. B. Moore, J. Chem. Phys., 1987, 86, 6000.

29 S. D. Peyerimhoff, M. Lewerenz and M. Quack, Chem. Phys. Lett., 1984, 109, 567; J. E. Baggott, M-C. Chuang, R. N. Zare, H-R. Dübal and M. Quack, J. Chem. Phys., 1985, 82, 1186; see also M. Lewerenz and M. Quack, J. Chem. Phys., submitted for publication for new interesting results.

30 K. Okuyama, N. Mikami and M. Ito, J. Phys. Chem., 1985, 89, 5617.

31 L. Halonen, M. S. Child and S. Carter, Mol. Phys., 1982, 47, 1097.

32 G. D. Carney, L. L. Sprandel and C. W. Kern, Adv. Chem. Phys., 1978, 37, 305; R. B. Gerber and M. A. Ratner, Chem. Phys. Lett., 1979, 68, 195.

33 D. W. Noid, M. L. Koszykowski and R. A. Marcus, J. Chem. Phys., 1977, 67, 404; M. L. Koszykowski, D. W. Noid and R. A. Marcus, J. Phys. Chem., 1982, 86, 2113; D. M. Wardlaw, D. W. Noid and R. A. Marcus, J. Phys. Chem., 1984, 88, 536.

34 T. Oka, Phys. Rev. Lett., 1980, 45, 531; T. Amano and J. K. G. Watson, J. Chem Phys., 1984, 81, 2869; for dissociation, A. Carrington and R. A. Kennedy, J. Chem. Phys., 1984, 81, 91.

35 B. G. Sumpter and D. W. Noid, Chem. Phys. Lett., 1986, 126, 181; D. W. Noid, S. K. Knudson, M. L. Koszykowski and R. L. Renka, J. Phys. Chem., 1986, 90, 6135; S. K. Knudson, D. W. Noid and M. L. Koszykowski, J. Phys. Chem., 1987, 91, 2763.

36 S. K. Knudson and D. W. Noid, Chem. Phys. Lett., 1988, 145, 16.

37 D. W. Noid and R. A. Marcus, J. Chem. Phys., 1975, 62, 2119; D. W. Noid, M. L. Koszykowski and R. A. Marcus, Annu. Rev. Phys. Chem., 1981, 32, 267.

38 D. W. Noid and R. A. Marcus, J. Chem. Phys., 1986, 85, 3305.

39 W. Eastes and R. A. Marcus, J. Chem. Phys., 1974, 61, 4301.

40 D. W. Noid, B. Brooks, S. K. Gray and S. L. Marple, J. Phys. Chem., in press; D. W. Noid and S. K. Gray, Chem. Phys. Lett., in press.

41 E.g. K. V. Reddy and M. J. Berry, Chem. Phys. Lett., 1979, 66, 223; T. M. Ticich, T. R. Rizzo, H-R. Dübal and F. F. Crim, J. Chem. Phys., 1986, 84, 1508; N. F. Scherer and A. H. Zewail, J. Chem. Phys., $1987,87,97$.

42 L. R. Khundkar, J. L. Knee and A. H. Zewail, J. Chem. Phys., 1987, 87, 77.

43 J. C. Stephenson, S. E. Bialkowski, D. S. King, E. Thiele, J. Stone and M. F. Goodman, J. Chem. Phys., $1981, \mathbf{7 4}, 3905$.

44 W. B. Miller, S. A. Safron and D. R. Herschbach, J. Chem. Phys., 1972, 56, 3581; J. M. Farrar and Y. T. Lee, ACS Symp. Ser., 1978, 66, 191.

45 M. G. Moss, M. D. Ensminger, G. M. Stewart, D. Mordaunt and J. D. McDonald, J. Chem. Phys., $1980,73,1256$.

46 H. W. Cruse, P. J. Dagdigian and R. N. Zare, Faraday Discuss. Chem. Soc., 1973, 55, 277.

47 B. A. Ruf and W. H. Miller, J. Chem. Soc., Faraday Trans. 2, 1988, 84, 1523.

48 D. M. Wardlaw and R. A. Marcus, Chem. Phys. Lett., 1984, 110, 230; J. Chem. Phys., 1985, 83, 3462; J. Phys. Chem., 1986, 90, 5383; 1987, 91, 4864; Adv. Chem. Phys., 1987, 70, Part I, 231; S. J. Klippenstein and R. A. Marcus, J. Chem. Phys., 1987, 87, 3410; J. Phys. Chem., 1988, 92, in press.

49 R. A. Marcus, Chem. Phys. Lett., 1988, 144, 208.

50 S. J. Klippenstein, L. R. Khundkar, A. H. Zewail and R. A. Marcus, J. Phys. Chem., to be submitted.

51 W. L. Hase, Chem. Phys. Lett., 1987, 139, 389 and references therein; M. Quack and J. Troe, Ber. Bunsenges. Phys. Chem., 1980, 81, 329; G. P. Smith and D. M. Golden, Int. J. Chem. Kinet., 1978, 10, 489; S. W. Benson, Can. J. Chem., 1983, 61, 881; R. A. Marcus, J. Phys. Chem., 1986, 90, 5385.

52 S. J. Klippenstein and R. A. Marcus, J. Phys. Chem., 1988, 92, in press.

53 C. Wittig, I. Nadler, H. Reisler, M. Noble, J. Catanzarite and G. Radhakrishnan, J. Chem. Phys., 1986, 85,1710 and references therein.

54 L. J. Butler, T. M. Ticich, M. D. Likar and F. F. Crim, J. Chem. Phys., 1986, 85, 2331, and references therein.

55 W. H. Green, I-C. Chen and C. B. Moore, Ber. Bunsenges. Phys. Chem., in press.

56 W. J. Chesnavitch, L. Bass, T. Su and M. T. Bowers, J. Chem. Phys., 1981, 74, 2228.

57 E.g. C. J. Cobos and J. Troe, J. Chem. Phys., 1985, 85, 1010.

58 M. Quack and J. Troe, Ber. Bunsenges. Phys. Chem., 1974, 78, 240; 1977, 81, 329; J. Troe, J. Chem. Phys., 1983, 79, 6017. 
59 R. E. Wyatt, Adv. Chem. Phys., in press.

60 G. A. Voth and R. A. Marcus, J. Chem. Phys., 1986, 84, 2254; S. J. Klippenstein, G. A. Voth and R. A. Marcus, J. Chem. Phys., 1986, 85, 5019.

61 W. Nadler and R. A. Marcus, J. Chem. Phys., 1987, 86, 6982; Chem. Phys. Lett., 1988, $144,509$.

62 E. B. Stechel and E. J. Heller, Annu. Rev. Phys. Chem., 1984, 35, 563.

63 S. M. Lederman, S. J. Klippenstein and R. A. Marcus, Chem. Phys. Lett., in press; S. M. Lederman and R. A. Marcus, J. Chem. Phys., 1988, 88, 6312.

64 J. V. Tietz and S-I. Chu, Chem. Thys. Lett., 1983, 101, 446; J. Chang and R. E. Wyatt, Chem. Phys. Lett., 1985, 121, 307; J. Chem. Phys., 1986, 85, 1826, 1840.

65 J. Chang, N. Moiseyev and R. E. Wyatt, J. Chem. Phys., 1986, 84, 4997.

66 R. A. Marcus, J. Chem. Phys., 1966, 45, 4493, 4500; 1968, 49, 2617.

67 W. H. Miller, N. C. Handy and J. E. Adams, J. Chem. Phys., 1980, 72, 99.

68 P. G. Hipes and A. Kuppermann, J. Chem. Phys., 1986, 84, 5962; R. T. Pack and G. A. Parker, J. Chem. Phys., 1987, 87, 3888. For additional discussion of hyperspherical coordinate systems see also C. A. Mead, J. Chem. Phys., 1980, 72, 3839; R. B. Johnson, J. Chem. Phys., 1980, 73, 5051; 1983, 79, 1906.

69 J. P. Dwyer, Ph.D. Thesis (California Institute of Technology, 1975); A. Kuppermann, J. A. Kaye and J. P. Dwyer, Chem. Phys. Lett., 1980, 74, 257; R. A. Marcus, Faraday Symp. Chem. Soc., 1975, 10, 60; J. Manz and J. Römelt, Chem. Phys. Lett., 1981, 81, 179.

70 V. K. Babamov and R. A. Marcus, J. Chem. Phys., 1981, 74, 1790.

71 J. A. Kaye and A. Kuppermann, Chem. Phys. Lett., 1981, 77, 573; P. G. Hipes and A. Kuppermann, J. Chem. Phys., 1986, 90, 3630.

72 V. K. Babamov, V. Lopez and R. A. Marcus, Chem. Phys. Lett., 1983, 101, 507; 1984, 81, 3962.

73 V. K. Babamov, V. Lopez and R. A. Marcus, J. Chem. Phys., 1983, 78, 5621; 1984, 81, 4182.

74 This topic is discussed, for example, in the following books: A. J. Lichtenberg and M. A. Lieberman, Regular and Stochastic Motion (Springer-Verlag, New York, 1983); V. I. Arnold and A. Avez, Ergodic Problems of Classical Mechanics (Benjamin, New York, 1968); R. Abraham and J. E. Marsden, Foundations of Mechanics (Benjamin-Cummings, Reading, 2nd edn, 1978); C. L. Siegel and J. K. Moser, Lectures on Celestial Mechanics (Springer-Verlag, New York, 1971). Numerous articles are given in Ann. N. Y. Acad. Sci., 1980, 357 and AIP Conf. Proc., 1978, 46.

75 G. Contopoulos, Z. Astrophys., 1960, 49, 1960; A. Ollongren, Bull. Astron. Inst. Neth., 1962, 16, 241; G. Contopoulos, Astron. J., 1963, 68.

76 Recent reviews include N. C. Handy, in Semiclassical Methods in Molecular Scattering and Spectroscopy, ed. M. S. Child (Reidel, Boston, 1980), p. 297; D. W. Noid, M. L. Koszykowski and R. A. Marcus, Annu. Rev. Phys. Chem., 1981, 32, 267; D. W. Noid and R. A. Marcus, ref. (38); G. S. Ezra, C. C. Martens and L. E. Fried, J. Phys. Chem., 1987, 91, 3721, among others.

77 G. Contopoulos, Astron. J., 1971, 76, 147; G. Contopoulos, Ann. N. Y. Acad. Sci., 1987, 497, 1.

78 C. Jaffe and W. P. Reinhardt, J. Chem. Phys., 1982, 77, 5191; R. Shirts and W. P. Reinhardt, J. Chem. Phys., 1982, 77, 5204.

79 B. V. Chirikov, Phys. Rep., 1979, 52, 263.

80 R. A. Marcus, Ann. N. Y. Acad. Sci., 1980, 357, 169; R. A. Marcus, Faraday Discuss. Chem. Soc., 1983, 75, 103; D. W. Noid, M. L. Koszykowski and R. A. Marcus, J. Chem. Phys., 1983, 78, 4018; T. Uzer, D. W. Noid and R. A. Marcus, J. Chem. Phys., 1983, 79, 4412.

81 R. A. Marcus, in Horizons of Quantum Chemistry, ed., K. Fukui and B. Pullman (Reidel, Dordrecht, 1980), p. 107; D. W. Noid, M. L. Koszykowski and R. A. Marcus, ref. (76).

82 I. C. Percival, Adv. Chem. Phys., 1979, 36, 1.

83 M. V. Berry, Ann. Phys. N. Y., 1981, 131, 163

84 E. V. Shuryak, Sov. Phys. JETP, 1976, 44, 1070; M. V. Berry, AIP Conf. Proc., 1978, 46, 115; K. G. Kay, J. Chem. Phys., 1980, 72, 5955.

85 D. Bensimon and L. P. Kadanoff, Ann. N. Y. Acad. Sci., 1987, 797, 110, and references therein; L. L. Gibson, G. C. Schatz, M. A. Ratner and M. J. Davis, J. Chem. Phys., 1987, 86, 3262.

86 Articles in Chaotic Behavior in Quantum Systems., ed. G. Casati, NATO ASI Series (Plenum, New York, 1983), vol. 120. 